\title{
Interferences in the density of two Bose-Einstein condensates consisting of identical or different atoms
}

\author{
L. S. Cederbaum ${ }^{1}$, A. I. Streltsov ${ }^{1}$, Y. B. Band ${ }^{2}$, and O. E. Alon ${ }^{1}$ \\ ${ }^{1}$ Theoretical Chemistry, Heidelberg University, Im Neuenheimer Feld 229, \\ 69120 Heidelberg $\&$ Max-Planck Institute for Nuclear Physics, \\ Saupfercheckweg 1, 69117 Heidelberg, Germany and \\ ${ }^{2}$ Department of Chemistry, Ben-Gurion University of the Negev, Beer-Sheva 84105, Israel
}

\begin{abstract}
The density of two initially independent condensates which are allowed to expand and overlap can show interferences as a function of time due to interparticle interaction. Two situations are separately discussed and compared: (1) all atoms are identical and (2) each condensate consists of a different kind of atoms. Illustrative examples are presented.

PACS numbers: PACS numbers: 05.30.Jp, 03.65.-w, 03.75.-b, 03.75.Mn
\end{abstract}


The investigation of interferences between particles is one of the most basic tools to learn on the nature of quantum gases. Interferences attracted much attention in particular in the case of Bose-Einstein condensates (BECs) both from the theoretical and experimental sides, see, e.g., [1, 2, 3, 4, 4, 5, 6, 7]. In a popular set up studied, identical atoms are produced in two traps which we may call the left and right traps and which are separated by a barrier. By removing the traps and the barrier between them, the atoms expand freely and can overlap. In experiment, the photographs obtained show spectacular interference fringes [1, 2].

The interference of two parts of a single coherent condensate is by now well understood, see, e.g., [4, 8, 9]. On the other hand, relatively little is known on the interference of two initially independent (i.e., fragmented) BECs, except for the case of non-interacting particles [3, 5, 6]. Fragmented BECs can be produced using a barrier between the two traps which is so high and broad that tunneling between them is negligible.

In the available experiments, the atoms are prepared in a double-well trap potential and it is not generally proven whether the atoms form a coherent BEC, a fragmented BEC, or a combination thereof. However, it is feasible nowadays to produce in the lab two spatially separated, initially independent BECs, see, e.g., [10], and this allows for experiments with definitely fragmented BECs. Apart from its importance as a fundamental problem, the solution of the problem of interference of two initially independent condensates is thus also of practical relevance.

In the scenario of two initially independent BECs the initial state of the many-body system before removing the traps reads

$$
|\Psi\rangle=\left(N_{L} ! N_{R} !\right)^{-1 / 2}\left(b_{L}^{\dagger}\right)^{N_{L}}\left(b_{R}^{\dagger}\right)^{N_{R}}|v a c\rangle, \quad N_{L}+N_{R}=N,
$$

where the $b_{L}^{\dagger}$ and $b_{R}^{\dagger}$ are the usual creation operators for bosons in the left and right traps, respectively, which contain definite numbers $N_{L}$ and $N_{R}$ of atoms in them. After removing the traps, the state $|\Psi\rangle$ is no longer an eigenstate of the system's Hamiltonian $H_{0}$ and expands in space as a function of time. The time-dependent density, i.e., the expectation value of the density operator $\hat{\rho}(x)$ as a function of time becomes [7]

$$
\rho(x, t) \equiv\langle\Psi(t)|\hat{\rho}(x)| \Psi(t)\rangle=N_{L}\left|\Phi_{L}(x, t)\right|^{2}+N_{R}\left|\Phi_{R}(x, t)\right|^{2},
$$

where the $\Phi_{L, R}(x, t)$ are the single-atom states corresponding to $b_{L, R}(t)=$ $\exp \left(i H_{0} t\right) b_{L, R} \exp \left(-i H_{0} t\right)$. Obviously, the density is a sum of the individual densities of the two condensates and does not exhibit an interference term. 
We would like to draw attention to the fact that the literature result (2) has been obtained under the assumption that atoms belonging to the two different BECs do not interact with each other. Very recently it has been demonstrated that, in the presence of interaction, the density $\rho(x, t)$ does show an interference term [11, 12]:

$$
\rho(x, t)=\rho_{L L}(x, t)+\rho_{R R}(x, t)+\rho_{L R}(x, t),
$$

where $\rho_{L L}$ and $\rho_{R R}$ are the densities of the expanding separated BECs as if the two BECs do not communicate, and $\rho_{L R}$ is the change of the density due to the interaction between them. The terms contributing to $\rho_{L L}\left(\rho_{R R}\right)$ contain only $b_{L}\left(b_{R}\right)$ and $b_{L}^{\dagger}\left(b_{R}^{\dagger}\right)$ operators, e.g., $b_{L}^{\dagger} b_{L}^{\dagger} b_{L} b_{L}$, and those contributing to $\rho_{L R}$ contain only mixed products, e.g., $b_{L}^{\dagger} b_{R}^{\dagger} b_{L} b_{R}$. The finding (3) has many consequences. In particular, the corresponding interference structures remain after the statistical averaging over many experimental runs. Of course, as $\rho(x, t)$ changes in time, the average must be carried out at the same value of $t$.

To derive (3) the full Hamiltonian $H=H_{0}+V$ of the system after removing the traps including the particle-particle interaction $V$ has been taken into account. For the ease of presentation, we employ the widely used contact interaction $V\left(x, x^{\prime}\right)=\lambda \delta\left(x-x^{\prime}\right)$, where $\lambda$ is proportional to the s-wave scattering length [7, 13]. Of course, any other interparticle interaction can be used as well. As usual, $H_{0}$ describes the motion of the free atoms. Starting from $H=H_{0}+V$ and the initial many-body state (1), we have obtained the exact result for $\rho(x, t)$ up to first order in the particle-particle interaction strength $\lambda$. The corresponding expression is somewhat lengthy and is not given here, but can be found in [12]. Let us briefly mention properties of this result. Clearly, the interference term $\rho_{L R}$ vanishes for $t \rightarrow 0$. Furthermore, $\rho_{L R}(x, t)$ vanishes as expected if the atoms do not interact with each other $(\lambda \rightarrow 0)$. The interference term $\rho_{L R}(x, t)$ is enhanced by the product $N_{L} N_{R}$ of the numbers of atoms in the two initial BECs.

The above discussions make clear that the interaction between the particles gives rise to an interference term in the density of two initially independent BECs of identical bosons. Before presenting a numerical example we go one step further and pose the question whether we can formulate a mean-field theory which reproduces exactly the many-body small $\lambda$ result mentioned above. Such a theory would open the door for real applications. The usual mean-field theory leads to the well-known and widely used Gross-Pitaevskii equation which reproduces exactly the density of BECs in a coherent state in the weak interaction 
limit [7, 13]. Clearly, this equation is inapplicable to BECs in fragmented states (11). For fragmented states a more general multi-orbital mean-field theory has been recently derived [14]. In the present scenario two orbitals are involved and the respective time-dependent mean-field $[\mathrm{TDMF}(2)]$ takes on the appearance (for the general derivation of TDMF, see [15]):

$$
\begin{aligned}
i \dot{\psi}_{L} & =\mathcal{P}\left[\hat{h}+\lambda\left(N_{L}-1\right)\left|\psi_{L}\right|^{2}+2 \lambda N_{R}\left|\psi_{R}\right|^{2}\right] \psi_{L}, \\
i \dot{\psi}_{R} & =\mathcal{P}\left[\hat{h}+\lambda\left(N_{R}-1\right)\left|\psi_{R}\right|^{2}+2 \lambda N_{L}\left|\psi_{L}\right|^{2}\right] \psi_{R}
\end{aligned}
$$

where the initial conditions are $\psi_{L, R}(x, t=0) . \hat{h}$ is the usual one-particle Hamiltonian (in our scenario just the kinetic energy operator $\left.-\frac{1}{2 m} \frac{\partial^{2}}{\partial x^{2}}\right)$ and $\mathcal{P}=1-\left|\psi_{L}\right\rangle\left\langle\psi_{L}|-| \psi_{R}\right\rangle\left\langle\psi_{R}\right|$ is a projector which ensures orthonormalization of the orbitals $\psi_{L}$ and $\psi_{R}$ [15]. In TDMF(2) the density can be expressed by $\rho(x, t)=N_{L}\left|\psi_{L}(x, t)\right|^{2}+N_{R}\left|\psi_{R}(x, t)\right|^{2}$. Indeed, it can be shown [12] that the $\operatorname{TDMF}(2)$ exactly reproduces the many-body result in the weak interaction limit.

In the following we apply the $\operatorname{TDMF}(2)$ theory (44). For coherent states the timedependent Gross-Pitaevskii equation, which is exact in the weak interaction limit, has been demonstrated in many cases to be applicable for intermediate and stronger interactions [7, 13]. Similarly, there is reason to expect that for fragmented states the TDMF theory, which has been proven to be exact in the weak interaction limit [12], is applicable well beyond this limit. We mention that $\operatorname{TDMF}(1)$ is nothing but the time-dependent Gross-Pitaevskii equation.

We consider harmonic traps centered at $\pm x_{0}$, each containing a BEC with interaction $\lambda=0.1$. At $t=0$ these traps are removed. As initial conditions $\psi_{L, R}(x, 0)$ we choose the respective solutions of the stationary Gross-Pitaevskii equation at this $\lambda$ to account for the interaction when the harmonic traps are released. In Fig. 1 the density $\rho(x, t)$ computed using the $\operatorname{TDMF}(2)$ equations is shown as a function of time. As seen in the figure, at $t=0$ the density consists of two separated distributions centered at $\pm x_{0}$. The traps are removed at this time and the distributions start to broaden and to overlap. At about $t=3$ one begins to see impact of the interference term in the density which becomes strongly pronounced as time proceeds.

We see that the density of two initially independent condensates which are allowed to overlap can show interference effects in the presence of interparticle interaction. The physics 
of so called fragmented states, like the state in Eq. (1), is generally very different from that of coherent states [16]. Coherent states of condensates have been extensively studied, mostly in the framework of the Gross-Pitaevskii equation [7, 13]. A BEC in a coherent state can exhibit interference fringes even in the absence of interaction [7, 8, 9, 17, 18]. Take, for instance, the coherent state $\left|\Psi^{c o h}\right\rangle=(N !)^{-1 / 2}\left(b^{\dagger}\right)^{N}|v a c\rangle$ with $b^{\dagger}=\left(b_{L}^{\dagger}+b_{R}^{\dagger}\right) / \sqrt{2}$. This immediately leads to $\rho^{c o h}(x, t)=\frac{N}{2}\left|\Phi_{L}(x, t)+\Phi_{R}(x, t)\right|^{2}$ in the absence of interaction between the atoms, and hence to the interference term $\rho_{L R}^{c o h}(x, t)=N R e\left(\Phi_{L}^{*} \Phi_{R}\right)$. For expanding Gaussians with initial width $2 a$ located at $\pm x_{0}$, the oscillatory part of $\rho_{L R}^{c o h}$ is simply given by $\cos [K(t) x]$ with $K(t)=8 x_{0}(t / m) /\left(a^{4}+4 t^{2} / m^{2}\right)$. This interference term is qualitatively different from that arising due to the interaction between the particles. Another important difference between $\rho_{L R}^{c o h}(x, t)$ and $\rho_{L R}(x, t)$ worth mentioning is that the former depends on the relative phase between $\Phi_{L}$ and $\Phi_{R}$, while the latter does not depend on this phase.

Whether in an experiment the initial state is coherent or fragmented depends on the experimental conditions. It is beyond the scope of this work to argue whether or not the initial state in the currently available experiments on interference is fragmented. It is also not our intention to take side in the ongoing debate on whether these experiments detect the density or higher-order correlation functions, although we tend to share the opinion of some researchers see, e.g., [4, 8, 9, 17], that the density is measured. What we can state, is that if one measures the density of two freely expanding initially independent BECs, it will only show interferences in the presence of interaction. This leads to the following proposal for an experiment which makes use of the fact that nowadays one can vary the strength of the interaction between the atoms [19, 20]. Two measurements are necessary. If the measurement with interaction shows interferences which disappear upon measuring with the interaction turned off, then (a) the initial state was a fragmented state and (b) the interaction is responsible for the interferences.

Until now the indistinguishability of the atoms has been considered a precondition for interference effects. In experiments on interferences one often starts with a single coherent BEC made of identical bosons and produces two BECs by ramping up a barrier. Interferences are then observed after removing the traps and the barrier. Nearly all theoretical works on interferences in BECs rely on the property of coherence of the whole system consisting of identical particles. Even the two very recent works [11, 12] which discuss interferences of identical independent BECs due to interaction between the particles have assumed that 
indistinguishability is necessary. We show below that this is unnecessary.

As above, we consider two initially independent BECs in an initial state like in (1). Now, however, each of these two BECs is made of a different kind of atoms. For simplicity we call them "left" and "right" atoms, and assign to them the creation operators $b_{L}^{\dagger}$ and $b_{R}^{\dagger}$ in (11). As usual, the Hamiltonian $H=H_{0}+V$ now contains three interactions terms $V=$ $V_{L}+V_{R}+V_{L R}$ accounting for the interaction between the "left" particles $\left[V_{L}=\lambda_{L} \delta\left(x-x^{\prime}\right)\right]$, between the "right" particles $\left[V_{R}=\lambda_{R} \delta\left(x-x^{\prime}\right)\right]$, and between the particles of both kinds $\left[V_{L R}=\lambda_{L R} \delta\left(x-x^{\prime}\right)\right]$, respectively. Using the same basic techniques as in [12] for identical particles, we obtain equation (3) for the density also in the present case of distinguishable BECs. The density contains a term $\rho_{L R}$ due to the interaction $V_{L R}$ between the particles of the two BECs. Moreover, the analytic expression for $\rho$ in the weak interaction limit is very similar to that in [12] for identical particles.

In contrast to the case of indistinguishable bosons, where the time-dependent mean-field equations for fragmented condensates have been derived very recently [15], time-dependent mean-field equations for mixtures of different bosons are well known [21, 22, 23]. For the present situation the latter read

$$
\begin{aligned}
i \dot{\psi}_{L} & =\left[\hat{h}_{L}+\lambda_{L}\left(N_{L}-1\right)\left|\psi_{L}\right|^{2}+\lambda_{L R} N_{R}\left|\psi_{R}\right|^{2}\right] \psi_{L}, \\
i \dot{\psi}_{R} & =\left[\hat{h}_{R}+\lambda_{R}\left(N_{R}-1\right)\left|\psi_{R}\right|^{2}+\lambda_{L R} N_{L}\left|\psi_{L}\right|^{2}\right] \psi_{R}
\end{aligned}
$$

where for simplicity we have used the same nomenclature for the orbitals $\psi_{L, R}(x, t)$ as for identical particles and the kinetic energies $\hat{h}_{L, R}=-\frac{1}{2 m_{L, R}} \frac{\partial^{2}}{\partial x^{2}}$ differ due to the possibly different masses of the left and right bosons. It is not surprising that (15) reproduce the exact result for the density in the weak interaction limit.

It is illuminating to briefly compare equations (5), which we call time-dependent coupled Gross-Pitaevskii [TDCGP(2)] equations, to the $\operatorname{TDMF}(2)$ for identical bosons (4). For identical bosons there are only a single mass $m$ and interaction strength $\lambda$, and a factor 2 appears in (41) due to the exchange of identical bosons. More importantly, TDMF(2) maintains the orthogonality of the orbitals $\psi_{L}$ and $\psi_{R}$ while TDCGP(2) does not.

To demonstrate that the density exhibits an oscillatory pattern also for interacting distinguishable condensates, we show a few examples computed via (5). For simplicity we put $m_{L}=m_{R}=m$. In our first example we choose $\lambda_{L R}=2 \lambda_{L}=2 \lambda_{R}=0.2$ which leads to the analogous scenario discussed in Fig. 1 for identical atoms. At $t=0$ the initial density is as 
in Fig. 1. At later times the density can evolve differently than in Fig. 1 only because the orbitals $\psi_{L}$ and $\psi_{R}$ do not have to be orthogonal to each other for distinguishable atoms. In Fig. 2 the density is shown for $t=8$ and compared with the analogous density obtained with (4) for identical particles. Both identical and distinguishable systems exhibit oscillatory structure but their differences are substantial. In the lower part of Fig. 2 we show the individual subdensities $N_{L}\left|\psi_{L}\right|^{2}$ and $N_{R}\left|\psi_{R}\right|^{2}$ at $t=8$ and remark that even a moderate overlap $\left\langle\psi_{L} \mid \psi_{R}\right\rangle$ can have a considerable impact on the oscillatory pattern of the density.

In our next example we choose $\lambda_{L}=\lambda_{R}=0$ and $\lambda_{L R}=0.2$, implying that the atoms in each of the left and right condensates do not interact, but those belonging to different condensates do. At $t=0$ we thus have two normalized Gaussians localized at the minima $\pm x_{0}$ of the harmonic traps. After removing the traps these Gaussians expand and overlap, and an oscillatory pattern develops. The result is shown for $t=22$ in the upper panel of Fig. 3. Until now the interactions studied were repulsive and we address the question whether an oscillatory structure can also arise for attractive interactions. To answer this question we investigate the same scenario but with $\lambda_{L R}=-0.2$. The result is depicted in the middle panel of Fig. 3. Remarkably, the oscillatory structure is even much more pronounced than for the repulsive interaction.

In our last example we ask whether an oscillatory pattern always evolves when interactions are present. We now choose $\lambda_{L}=\lambda_{R}=\lambda_{L R}=0.1$ and find no oscillations in the density up to quite long times. The density at $t=10$ is shown in the lower panel of Fig. 3. This result can be understood from (5). Assuming $m_{L}=m_{R}, \lambda_{L}=\lambda_{R}=\lambda_{L R}$ and $N_{L}=N_{R} \gg 1$, we notice that both equations (5) are actually equivalent as they depend only on the total density $\rho=N_{L}\left|\psi_{L}\right|^{2}+N_{R}\left|\psi_{R}\right|^{2}$. The analogous situation is not possible for identical particles because of the factor 2 appearing in (44) due to the exchange interaction.

The origin of the oscillations in the density is interference. Commonly, one attributes a phase difference to the appearance of interferences. This is particularly simple in the case of two parts of a coherent condensate which show interference effects when overlapping. One may attribute a phase to each of these parts. As discussed above for fragmented condensates of identical particles, the relative phase of the fragments is irrelevant in the context of interferences, and this is, of course, also the case for condensates made of different kinds of particles. In these situations, interparticle interactions are responsible for interference effects. Here, we may speak of interaction-assisted self-interference. Consider for simplicity two 
freely expanding, initially non-overlapping, different condensates with repulsive interaction between them. In each of these condensates the orbital has a phase which depends on $x$ and $t$. As usual for a freely expanding isolated condensate, its orbital and hence its phase are smoothly changing such that no interference occurs. Once the interacting condensates begin to overlap, atoms are decelerated in the overlapping region and the local phase changes there. At later times the changed part of the orbital is superposed with other expanding parts of the same coherent condensate and this leads to interferences. In the extreme case of infinitely strong repulsive interaction between the two condensates, each of the condensates is reflected from the other condensate as if it were an expanding hard wall. The interferences then arise from the superposition of the reflected and advancing parts of the orbital.

The theory presented here is easily extendable to any kind of interparticle interaction. It is also easily extendable to the case where one does not let the two BECs expand freely by removing the traps completely. One may, e.g., remove only the barrier and let the BECs expand in the new global trap. Since the interference structures depend on the interaction, a wealth of effects can be expected by varying the interaction, the form of the individual traps and the numbers $N_{L}$ and $N_{R}$ of the particles. In the case of distinguishable particles also the difference in masses $m_{L}, m_{R}$ and, most importantly, the intra- and inter-condensate interactions $\lambda_{L}, \lambda_{R}$ and $\lambda_{L R}$ enrich the possible range of interference phenomena.

\section{Acknowledgments}

Financial support and collaboration within the German-Israeli DIP project of the German Federal Ministry of Education and Research (BMBF) are gratefully acknowledged.

[1] M. R. Andrews et al., Science 275, 637 (1997).

[2] T. Schumm et al., Nature Physics 1, 57 (2005).

[3] J. Javanainen and S. M. Yoo, Phys. Rev. Lett. 76, 161 (1996).

[4] M. Naraschewski et al., Phys. Rev. A 54, 2185 (1996).

[5] Y. Castin and J. Dalibard, Phys. Rev. A 55, 4330 (1997).

[6] R. A. Hegstrom, Chem. Phys. Lett. 288, 248 (1998).

[7] A. J. Leggett, Rev. Mod. Phys. 73, 307 (2001). 
[8] A. Röhrl et al., Phys. Rev. Lett. 78, 4143 (1997).

[9] W.-M. Liu et al., Phys. Rev. Lett. 84, 2294 (2000).

[10] Y. Shin et al., Phys. Rev. Lett. 95, 170402 (2005).

[11] H. Xiong et al., New J. Phys. 8, 245 (2006).

[12] L. S. Cederbaum et al., cond-mat/0607556.

[13] L. Pitaevskii and S. Stringari, Bose-Einstein Condensation (Oxford University Press, Oxford, 2003).

[14] L. S. Cederbaum and A. I. Streltsov, Phys. Lett. A 318, 564 (2003).

[15] O. E. Alon et al., Phys. Lett. A, http://dx.doi.org/10.1016/j.physleta.2006.10.048.

[16] A. I. Streltsov et al., Phys. Rev. A 70, 053607 (2004); O. E. Alon and L. S. Cederbaum, Phys. Rev. Lett. 95, 140402 (2005).

[17] L. Pitaevskii and S. Stringari, Phys. Rev. Lett. 83, 4237 (1999).

[18] M. Albiez et al., Phys. Rev. Lett. 95, 010402 (2005).

[19] S. L. Cornish et al., Phys. Rev. Lett. 85, 1795 (2000).

[20] F. K. Fatemi et al., Phys. Rev. Lett. 85, 4462 (2000).

[21] H. Pu and N. P. Bigelow, Phys. Rev. Lett. 80, 1134 (1998).

[22] E. Timmermans, Phys. Rev. Lett. 81, 5718 (1998).

[23] P. Öhberg and L. Santos, Phys. Rev. Lett. 86, 2918 (2001). 

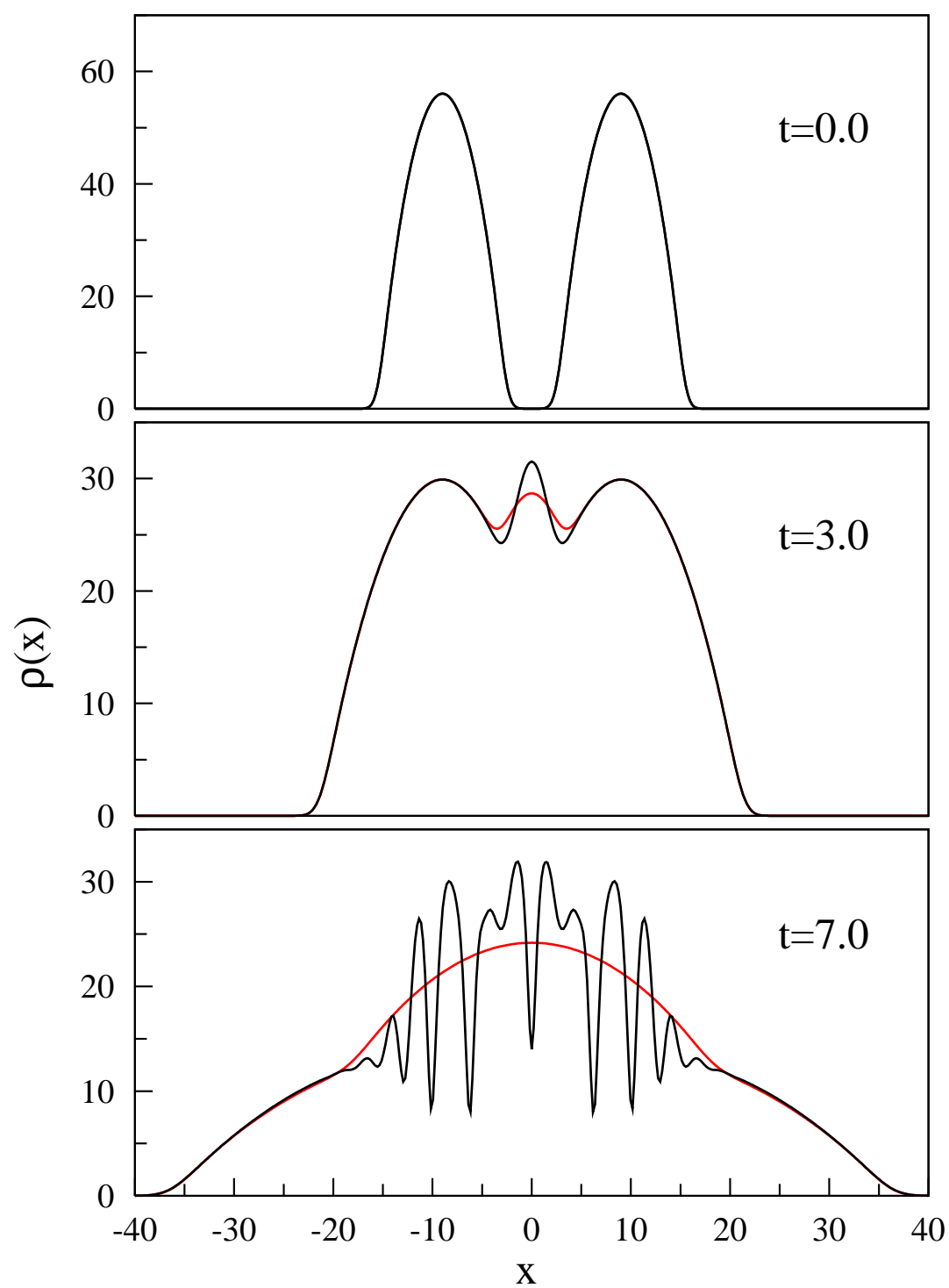

FIG. 1: (Color online) The density $\rho(x, t)$ of two condensates of 500 atoms each for $\lambda=0.1$ as a function of time computed with $\operatorname{TDMF}(2)$ (black) compared to the density $\rho_{L L}+\rho_{R R}$ of two BECs which do not interact with each other, each computed with the Gross-Pitaevskii equation (red). The quantities shown are dimensionless. For more details see text. 


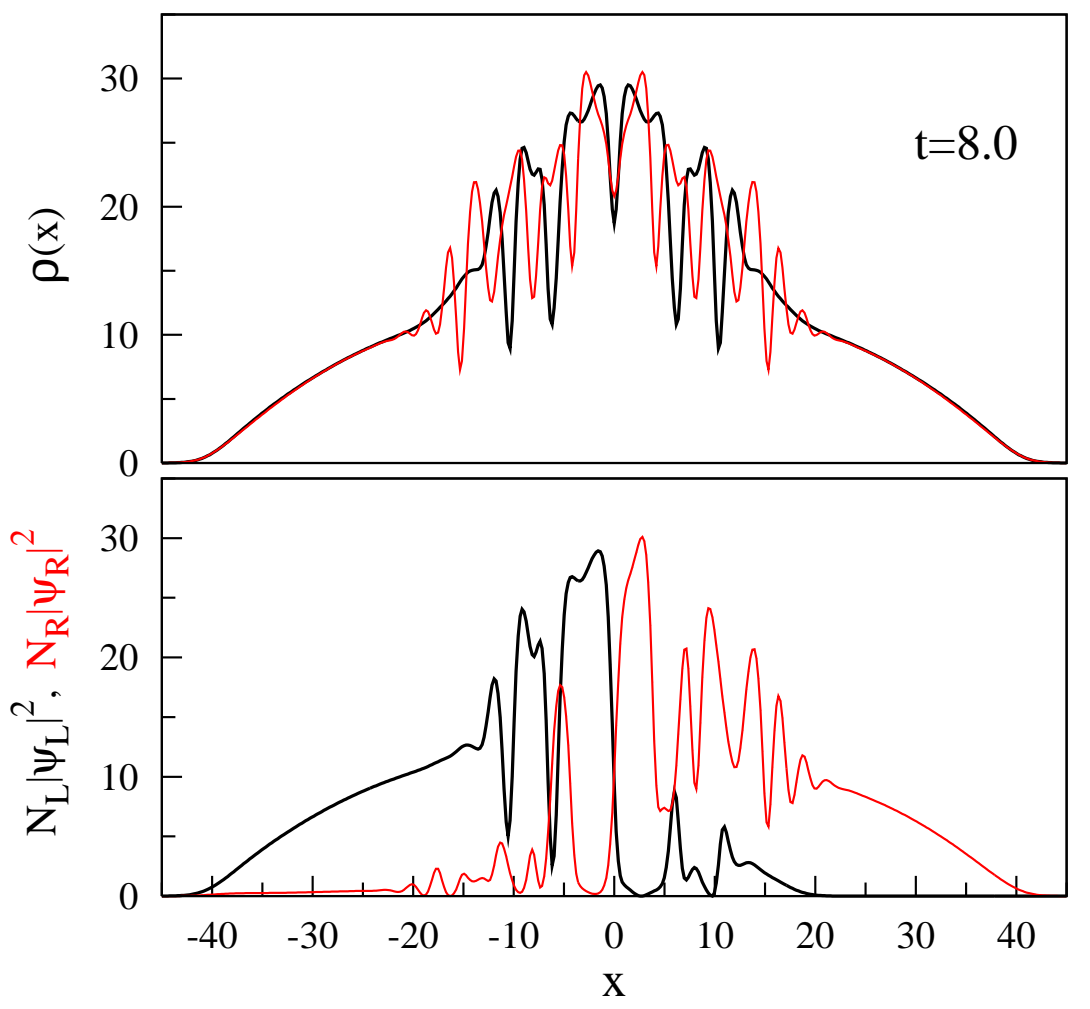

FIG. 2: (Color online) The density of two condensates each made of 500 atoms of a different kind computed with TDCGP(2) for $\lambda_{L}=\lambda_{R}=0.1$ and $\lambda_{L R}=0.2$ at $t=8$ (black). For comparison also the analogous result for identical particles computed with $\operatorname{TDMF}(2)$ (for details see Fig. 11) is shown (red). The lower panel shows the respective subdensities $N_{L}\left|\psi_{L}\right|^{2}$ for distinguishable atoms (black) and $N_{R}\left|\psi_{R}\right|^{2}$ for identical particles (red). 


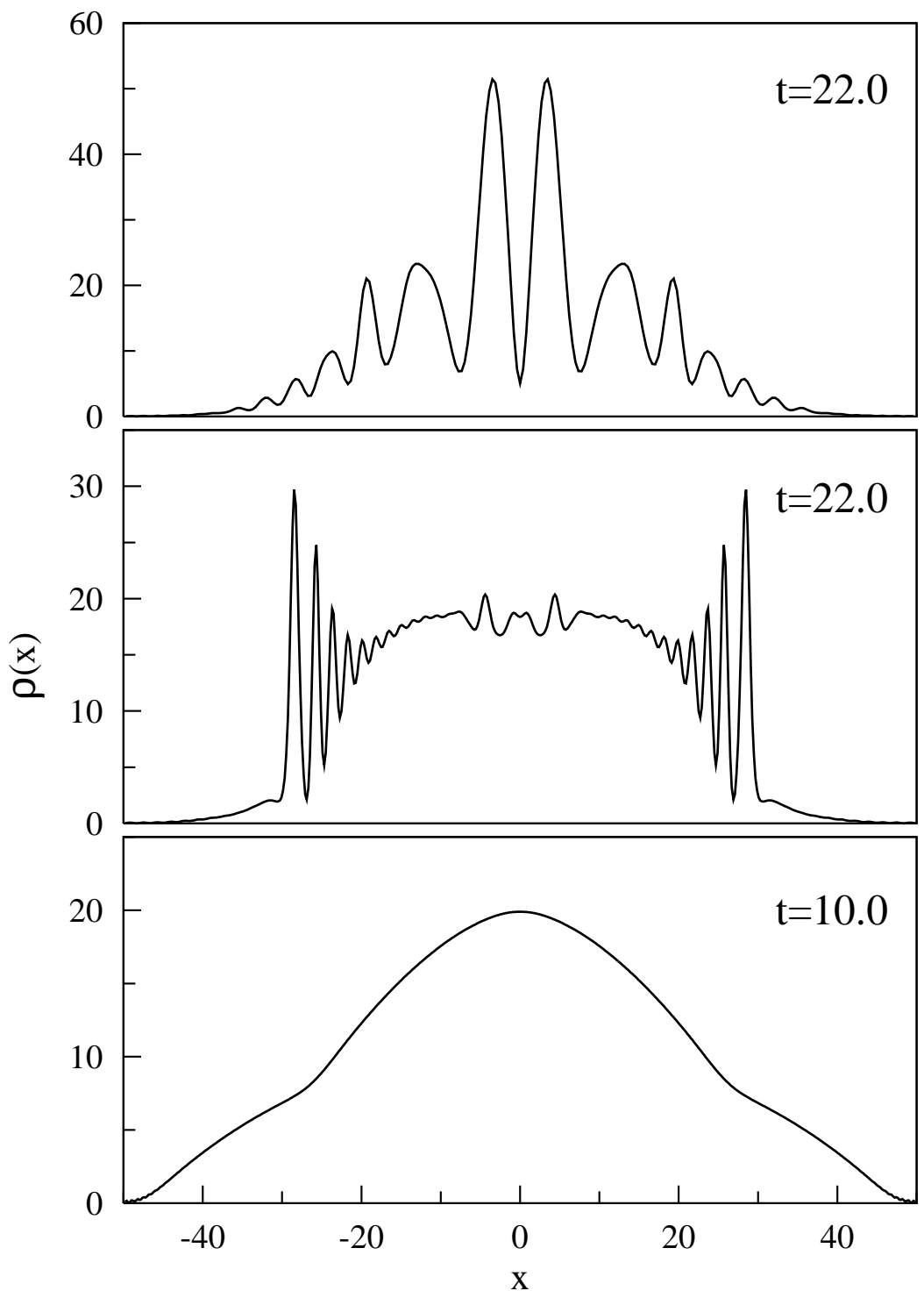

FIG. 3: The density of two condensates each made of 500 atoms of a different kind. Upper panel: repulsive interaction of the two condensates, $\lambda_{L}=\lambda_{R}=0, \lambda_{L R}=+0.2$. Middle panel: attractive interaction of the two condensates, $\lambda_{L}=\lambda_{R}=0, \lambda_{L R}=-0.2$. Lower panel: $\lambda_{L}=\lambda_{R}=\lambda_{L R}=0.1$. 